\title{
Prefactibilidad de una pequeña central hidroeléctrica en el Embalse del Sisga, Cundinamarca, Colombia*
}

\author{
Pre-feasibility Study of a Small Hydroelectric Plant in \\ El Sisga Reservoir, Cundinamarca, Colombia
}

\section{Nicolás Humberto Pacheco Garzón ${ }^{a}$, Jesús Ernesto Torres Quinterob}

\begin{abstract}
${ }^{a}$ Auxiliar de investigación programa de Ingeniería Ambiental, Universidad Libre, Colombia | nicolash-pachecog@unilibre.edu.co
${ }^{b}$ Ingeniero civil especializado en recursos hidráulicos con énfasis en hidrología. M. Sc. recursos hidráulicos. Docente investigador, Universidad Libre, Colombia. jesuse.torresq@unilibre.edu.co| https://orcid.org/0000-0003-1611-3707

* Artículo derivado del proyecto de investigación "Prefactibilidad de una pequeña central hidroeléctrica en el Embalse del Sisga, Cundinamarca", del grupo de investigación TECNOAMBIENTAL.
\end{abstract}

\section{RESUMEN}

Este trabajo tiene como objetivo establecer la viabilidad de instalar una pequeña central hidroeléctrica (PCH) en el Embalse del Sisga, dado que este cuenta con características topográficas e hidrológicas que pueden convertirlo en un generador de energía sin poner en riesgo el abastecimiento del recurso hídrico ni los activos ambientales de la zona. Los criterios para cumplir con este objetivo fueron establecidos al realizar estudios cartográficos, determinando el salto hidráulico y el recorrido de la tubería en el proyecto, junto con estudios hidrológicos, los cuales permitieron conocer el caudal de diseño y la capacidad energética. La selección de los componentes hidráulicos se llevó a cabo con base en los estudios anteriores, para finalmente estimar los impactos en las

Citation: Pacheco-Garzón, N. H. y Torres-Quintero, J. E. (2021). Prefactibilidad de una pequeña central hidroeléctrica en el Embalse del Sisga, Cundinamarca. Mutis, 11(2), 84-95. https://doi. org/10.21789/22561498.1785

Recibido: 10 de mayo de 2021. Aceptado: 30 de junio de 2021.

Copyright: $\odot 2021$. Pacheco-Garzón, N. H. y Torres-Quintero, J. E. (2021). This is an open-access article, which permits unrestricted use, distributions and reproduction in any medium, provided the original author and source are credited.

Competing Interests: The authors have no conflict of interest. categorías ambientales y en el componente socioeconómico y cultural en el área de influencia del proyecto. A partir de este estudio de prefactibilidad se pudo observar como una PCH en el Embalse del Sisga podría ser instalada de forma óptima y eficiente, sin incurrir en altos costos de instalación y operación, destacando además los beneficios de una PCH y los bajos impactos ambientales negativos de realizar este proyecto.

Palabras clave: fuentes de energía renovable, generación de energía hidroeléctrica, pequeña central hidroeléctrica. 


\section{ABSTRACT}

This work aims to establish the feasibility of installing a small hydroelectric power plant (SHPP) in the Sisga reservoir, given that this body of water has topographical and hydrological characteristics that can turn it into an energy generator without jeopardizing the supply of water resources or the environmental assets in this area. The criteria for meeting this objective were established after conducting cartographic studies that determined the hydraulic jump and the route of the pipe in the project, as well as hydrological studies that allowed us to know the design flow and energy capacity. The selection of hydraulic components was determined based on the results of the previous studies, to finally estimate the impacts on the environmental categories and on the cultural and socio-economic component in the area of influence of the project. From this pre-feasibility study, it was possible to observe how a SHPP in the Sisga reservoir could be installed optimally and efficiently without incurring in high installation or operational costs, highlighting the benefits of a SHPP and the low negative environmental impacts of this project.

Keywords: Renewable energy sources, hydroelectric power generation, small hydroelectric power plant.

\section{INTRODUCCIÓN}

El mundo busca cubrir de manera eficiente la demanda de energía eléctrica a través de tecnologías limpias, con el fin de disminuir la contaminación atmosférica generada por las centrales térmicas y las alteraciones ambientales derivadas de la construcción de nuevas centrales hidroeléctricas, además de evitar el desabastecimiento eléctrico como consecuencia de las irregularidades que sufren las centrales hidroeléctricas debido a fenómenos climáticos.

Una tecnología altamente conocida en el mundo son las pequeñas centrales hidroeléctricas $(\mathrm{PCH})$, caracterizadas por tener una gran variedad de capacidades instaladas y ser amigables con el medioambiente, además de tener una gran versatilidad de instalación (Fundación Solar, 2018). Las PCH brindan la ventaja de no requerir altas caídas ni grandes caudales de agua, por lo cual no generan altos impactos, como sucede con otros métodos de generación. Tanto es así que la Organización Mundial de las Naciones Unidas para el Desarrollo Industrial (ONUDI), en el Informe Mundial sobre el Desarrollo de la Pequeña Central Hidroeléctrica 2019, categoriza a las PCH como una tecnología madura y versátil, efectiva para proporcionar acceso a electricidad limpia y sostenible - tanto en los países en vía de desarrollo como en las naciones desarrolladas-, particularmente en las zonas rurales. Mediante el desarrollo de $\mathrm{PCH}$ muchos países han tomado medidas para aliviar la pobreza y aumentar el acceso a electricidad (ONUDI, 2019).
Las PCH tienen varios beneficios frente al uso de energías convencionales. En primer lugar, no utilizan combustibles fósiles para producir energía. Al respecto, el Grupo Intergubernamental de Expertos sobre el Cambio Climático, en su informe especial sobre fuentes de energía renovables y mitigación del cambio climático, explica cómo las energías alternativas disminuyen considerablemente los gases efecto invernadero (GEI):

Los valores medianos para el conjunto de las energías renovables están situados entre 4 y $46 \mathrm{~g}$ de $\mathrm{CO}_{2}$ eq/ $\mathrm{kWh}$, mientras que los combustibles de origen fósil están comprendidos entre 469 y $1.001 \mathrm{~g}$ de $\mathrm{CO}_{2}$ eq/ kWh (exceptuando las emisiones debidas a los cambios del uso de la tierra) (IPCC, 2011, p. 18).

Otra ventaja de las $\mathrm{PCH}$ es que no alteran en gran proporción los ecosistemas, en comparación con las grandes centrales hidroeléctricas. Esto se debe a que las PCH no necesitan inundar grandes áreas para poder producir energía, por lo que su funcionamiento no se ve afectado por fenómenos como El Niño, puesto que no requieren de altos volúmenes de agua para trabajar. Además, su versatilidad de instalación permite suministrar el servicio de energía a lugares de difícil acceso, ampliando el cubrimiento de la demanda energética.

A partir de los beneficios reportados de las PCH, estas tienen un alto grado de aceptación en el mundo. En algunos países que han ido implementado estas 
pequeñas centrales, se ha evidenciado la eficiencia en la producción de energía eléctrica:

En Euskadi, debido a que los ríos son de curso corto y no conducen caudales importantes, existen bastantes minicentrales hidráulicas. En algunos lugares de España hay problemas de escasez de agua y se han construido presas para riego. Posteriormente han sido aprovechadas para generar energía, y actualmente tenemos una fracción importante de energía hidroeléctrica instalada (Torres, 2010, p. 3).

En Sudamérica las PCH gozan de gran aceptación debido a que el continente tiene una enorme capacidad hídrica. Según UNIDO (United Nations Industrial Development Organization), en su informe Word Small Hydropower Development Report 2016:

La capacidad hidroeléctrica total instalada en América del Sur, incluyendo grandes, medianas y pequeñas instalaciones, es de aproximadamente 103 GW. Además, desde el 2013 la capacidad de las PCH ha incrementado un $14 \%$ y la capacidad de instalación ha incrementado un $31 \%$. Según este informe, las PCH tienen una capacidad instalada en Colombia de hasta $250 \mathrm{MW}$ y un potencial de $25.000 \mathrm{MW}$. Entre los Informes mundiales de desarrollo de pequeñas centrales hidroeléctricas de 2013 y 2016, la capacidad instalada aumentó en un $45 \%$ (UNIDO, 2016, p. 272).

Colombia cuenta con un gran potencial para instalar este tipo de tecnologías gracias a su capacidad hídrica y su relieve característico. Tanto así que con las $\mathrm{PCH}$ se inició el desarrollo del sistema eléctrico colombiano, dado que se evidenció que cerca de cada municipio había pequeñas caídas de agua que podrían ser aprovechables para la generación de energía (VélezÁlvarez, 2020). La implementación de las PCH en otros países, así como la capacidad y el potencial que tiene Colombia para instalar estas tecnologías, respalda la idea de que estas centrales contribuyen a mitigar las diferentes problemáticas antes mencionadas. Por estas razones, la presente investigación busca diseñar y sustentar la viabilidad de una pequeña central hidroeléctrica en el Embalse del Sisga, el cual puede contribuir a satisfacer la demanda de energía eléctrica del país y favorecer a Colombia en la lucha contra el desabastecimiento energético derivado del fenómeno El Niño.
El Embalse del Sisga cuenta con grandes aportes hídricos provenientes de diferentes fuentes. Según Colparques, este cuerpo de agua cuenta con una superficie de $148,2 \mathrm{~km}^{2}$ y una capacidad de diseño de $101,2 \mathrm{~mm}^{3}$, la cual ha ido variando debido a la colmatación natural resultado de 53 años de operación, por lo que su capacidad real es de $94,3 \mathrm{~mm}^{3}$, de los cuales $95 \%$ son volumen útil; en otras palabras, $89,6 \mathrm{~mm}^{3}$. Igualmente, estudios de la Corporación Autónoma Regional de Cundinamarca (CAR) han determinado una disminución en el área superficial del embalse, la cual se ha reducido de 653 a 637 hectáreas (Organización Colparques, 2020). Estos aportes hídricos demuestran la capacidad y viabilidad del embalse para producir energía eléctrica por medio de una $\mathrm{PCH}$.

A continuación, se presenta el estudio que busca evaluar los beneficios de instalar una PCH aprovechando las aguas del Embalse del Sisga.

\section{MATERIALES Y MÉTODOS}

\section{Descripción del sitio de estudio}

Como se muestra en la figura 1, el Embalse del Sisga se encuentra localizado en el municipio de Chocontá, Cundinamarca, a $55 \mathrm{~km}$ al norte de Bogotá en dirección a Tunja, Boyacá. El embalse se ubica a $2.674 \mathrm{~m}$ s. n. m. en la cuenca alta del río Bogotá, a la altura de los municipios de Chocontá, Sesquilé, Guatavita y Machetá. Sus coordenadas son $05^{\circ} 04^{\prime} \mathrm{N}$ y $73^{\circ} 44^{\prime} \mathrm{E}$.

Figura 1. Localización del Embalse del Sisga

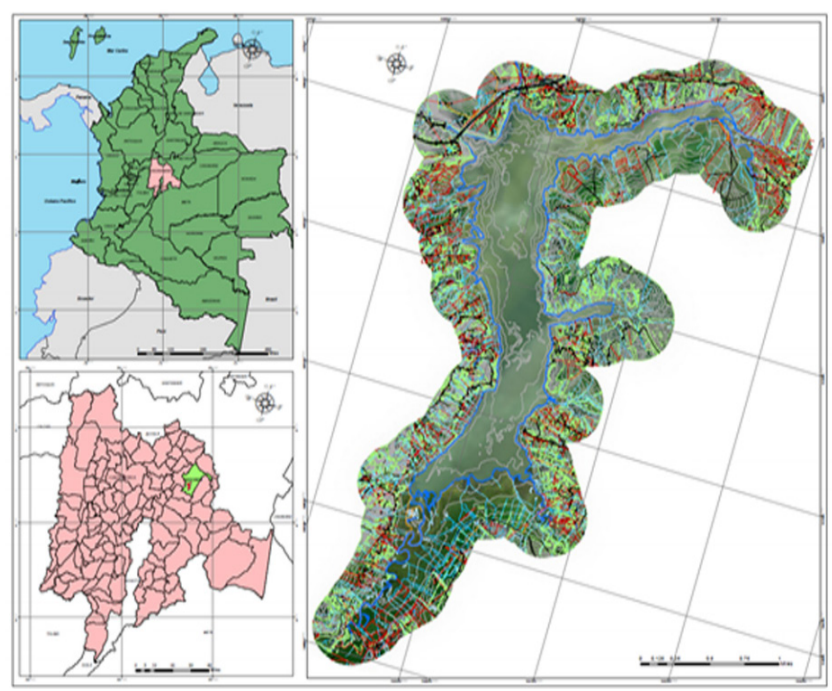

Fuente: manual de operación Embalse del Sisga. 
Según la CAR, en el manual de operación del Embalse del Sisga, el área de este cuerpo de agua se estima en $148,2 \mathrm{~km}^{2}$. El embalse tiene un aporte anual medio de $79,5 \mathrm{~mm}^{3}$, equivalente a un caudal medio de $2,52 \mathrm{~m}^{3} / \mathrm{s}$, proveniente principalmente del río San Francisco (64 $\%$, aproximadamente); es decir, 1,61 $\mathrm{m}^{3} / \mathrm{s}$. La quebrada Granadillo contribuye con $0,40 \mathrm{~m}^{3} / \mathrm{s}$, lo que equivale a $16 \%$ de la afluencia total. El caudal restante, que corresponde a $0,46 \mathrm{~m}^{3} / \mathrm{s}$, está constituido por el aporte de las lluvias y pequeñas cuencas no instrumentadas que drenan directamente al embalse.

La orografía de la zona donde se encuentra el embalse conforma un paisaje ondulado, cuya altura fluctúa entre 2.700 y $3.400 \mathrm{~m} \mathrm{~s}$. n. m., formando un valle enmarcado por la cordillera de Suratá, al noreste, y la cordillera de Peña Blanca, al suroccidente.

Las precipitaciones presentes en el área del embalse son producto de la vertiente de la Orinoquía, con influencia del régimen unimodal, que se caracteriza por presentar una temporada lluviosa entre mayo y agosto y un período seco entre diciembre y marzo, aumentando hacia el oriente y suroriente, en donde puede alcanzar hasta $1.300 \mathrm{~mm}$ (CAR, 2020). La cuenca hidrográfica en la cual se encuentra el embalse cuenta con calcificaciones climáticas frías, principalmente, y el embalse se encuentra situado en un clima frío semihúmedo. Gran parte de la cuenca contiene clima de páramo bajo semihúmedo (Consrocio Ecoforest Ltda., 2018).

\section{Datos}

Para realizar los diferentes estudios en el marco de la investigación fue necesario implementar elementos y herramientas de medición que permitieron la obtención de datos para su posterior análisis, los cuales se describen a continuación.

- Estación hidrometeorológica de la CAR: se obtuvieron los datos de caudales multianuales medios mensuales de la estación hidrometeorológica Embalse Sisga, que mide el caudal de descarga. Los datos suministrados son mediciones que van desde el año 1952 hasta enero de 2021.

- Planchas: se obtuvieron del Instituto Geográfico Agustín Codazzi (escala 1:25000), donde se podía localizar el Embalse del Sisga con su punto de descarga. Las planchas se adquirieron en formato físico, PDF y en Shapefile, con el fin de tener mayor versatilidad para trabajar sobre la información. Los números de las planchas son 209VIA y 209VIC.

\section{Metodología}

Para el desarrollo del presente estudio se establecieron 5 aspectos, para los cuales se aplicaron diversos métodos de análisis y dimensionamiento de los parámetros de importancia para una PCH en el área de estudio.

\section{Análisis topográfico}

El análisis topográfico consiste en realizar una estimación del salto de agua disponible. En los estudios de prefactibilidad de $\mathrm{PCH}$ es bastante común usar los mapas con curvas de nivel (Instituto de Ciencias Nucleares y Energías Alternativas, 1997), por lo que estos fueron empleados en el presente estudio. A partir de este análisis, fue posible establecer un recorrido viable para el recorrido del agua desde la salida del embalse hasta la casa de máquinas.

\section{Evaluación del recurso hidroenergético}

El análisis hidrológico consistió en determinar el caudal disponible para el proyecto y su capacidad de producir energía eléctrica. Para estimar el caudal de diseño se realizó una curva de duración de caudales (CDC), la cual brinda la probabilidad como un porcentaje de tiempo de todo el período de medición, en el que el caudal es igual o menor al caudal correspondiente a dicho porcentaje de tiempo (Ortiz-Flórez, 2011). En este sentido, la frecuencia relativa acumulativa corresponde a la duración en términos de porcentaje. Para calcular la frecuencia de cada rango simplemente se divide el número de ocurrencias entre el número total de aforos, como se observa en la siguiente ecuación:

$$
f r(\%)=\left(\frac{F}{N}\right) * 100 \quad(\text { Ec. } 1)
$$

Donde:

fr: frecuencia relativa

F: número de ocurrencias

$\mathrm{N}$ : número total de aforos.

Por su parte, el caudal medio se determina mediante la siguiente expresión. 


$$
Q m=\Sigma Q i * f r / 100
$$

Donde:

Qm: caudal medio

Qi: caudal

fr: frecuencia relativa.

A partir del desarrollo de la CDC fue posible realizar una curva de duración de potencias (CDP). Este análisis permite determinar el caudal que aporta mayor energía al sistema, es decir, cuántos kWh podría generar por año. Para trazar la CDP basta con convertir el eje de ordenadas de la CDC en eje de potencias, como se muestra en la siguiente expresión.

$$
P=\frac{\gamma * Q * H * \eta}{1000}
$$

Donde:

P: potencia

Ү: peso específico del agua

Q: caudal de diseño

$\mathrm{H}$ : altura neta

$\eta$ : eficiencia de la turbina.

\section{Selección de componentes hidráulicos y electromecánicos}

Esta investigación planteó la selección de la tubería de carga y la casa de máquinas, la cual está compuesta por la turbina y el generador.

\section{Tubería de carga}

El material que se seleccionó para la conducción del fluido es el acero. Por medio de la literatura se obtuvieron los coeficientes del conducto, datos necesarios para determinar variables que pueden generar inconvenientes en la conducción. Variables como la velocidad del fluido, la carga y la presión interna en la tubería fueron calculadas y consideradas para obtener un diámetro mínimo en la tubería (Yunus \& Cimbala, 2006). Las pérdidas por fricción fueron calculadas para determinar la caída neta del sistema por medio de la ecuación de Hazen-Williams, como se ve a continuación.

$$
h f=10,648\left(\frac{1}{C_{H W}}\right)^{1.852} * \frac{Q^{1,852}}{D^{4,871}} * L
$$

Donde:

Hf: pérdidas por fricción

$\mathrm{C}_{\mathrm{HW}}$ : coeficiente de fricción de Hazen-Williams

Q: caudal

D: diámetro

L: longitud de la tubería.

Adicional a lo relacionado anteriormente, se realizó el cálculo del diámetro económico que debería tener la tubería. Este valor es de gran importancia, dado que un diámetro menor supone menores costos de ejecución. Sin embargo, un diámetro menor implica un mayor costo energético debido a las pérdidas de fricción, por lo que los costos de explotación serán más altos. Por esta razón, el diámetro económico será aquel cuya suma de estas dos variables reporte el menor costo para el proyecto (Instituto de Ciencias Nucleares y Energías Alternativas, 1997).

\section{Casa de máquinas}

La casa de máquinas se compone principalmente del generador, la turbina y el tablero de controles, equipos seleccionados a partir del caudal y la altura de caída del agua. La casa de máquinas protege el equipamiento electromecánico de las adversidades climatológicas. El número, tipo y potencia de las turbinas, su disposición con respecto al canal de descarga, la altura de salto y la geomorfología del sitio condicionan el tamaño y la forma de la construcción (Riego, 2020). Algunas características de este tipo de turbinas se muestran en la tabla 1.

Tabla 1. Características principales de algunas turbinas hidráulicas

\begin{tabular}{|c|c|c|}
\hline Turbina & Caudal $\left(\mathrm{m}^{3} \mathbf{s}\right)$ & Caída $(\mathbf{m})$ \\
\hline Pelton & 0,05 a 50 & 30 a 1.800 \\
\hline Francis & 1 a 500 & 2 a 750 \\
\hline Kaplan & 100 & 5 a 80 \\
\hline
\end{tabular}

Fuente: Instituto de Ciencias Nucleares y Energías Alternativas (1997). 


\section{Análisis energético del sistema}

En este análisis se calculó el potencial energético, favoreciendo el sistema por medio de dos turbinas, con el objetivo de analizar las potencias máxima, media y firme; la última hace referencia a la potencia que puede generar el sistema operando con bajo caudal (Gugler Technology for Hydropower Plants, 2020).

Para realizar el estudio se calculó la potencia para cada caudal según su frecuencia, simulando el ingreso del agua a cada turbina, logrando evidenciar su rendimiento y capacidad de generación individualmente. La potencia máxima se calcula directamente con la caída neta y el caudal de diseño establecido en la CDC, como se realizó a través de la ecuación (3). La potencia media son los miles de kWh generados anualmente divididos por la cantidad de horas al año. Por su parte, la generación firme tiene en cuenta la potencia obtenida de los caudales más bajos en el año.

\section{Análisis ambiental}

Se realizó un análisis que consiste en relacionar la cantidad de $\mathrm{CO}_{2}$ que se evitaría al producir energía por medio de una $\mathrm{PCH}$, en comparación con una central termoeléctrica. Según la Unidad de Planeación Minero-Energética, producir $1 \mathrm{MW}$ de potencia por medio de estas centrales genera alrededor de 0,367 $\mathrm{t} \mathrm{CO}_{2}$ por año (UPME, 2020). Al remplazar la forma de generación de energía por medio de un medio alternativo a la quema de combustible, el proyecto genera impactos positivos en el ambiente. Esto se debe también a que las plantas termoeléctricas producen 30,7 $\%$ de la energía eléctrica del país (Acolgen, 2019), ocasionando un fuerte impacto en el ambiente.

Gracias al análisis energético realizado se determinó la producción de energía anual en el sistema. Con este dato es posible calcular con una sencilla relación cuánto $\mathrm{CO}_{2}$ sería producido por la quema de combustibles fósiles. La siguiente equivalencia muestra como fue calculado el $\mathrm{CO}_{2}$ producido.

$$
\text { TonCO }_{2}=\frac{0.367 \mathrm{TonCO}_{2} * P A(\mathrm{Mw}\}}{1(\mathrm{Mw})}
$$

Donde:

PA: producción anual de energía.

\section{RESULTADOS}

A continuación se muestran los resultados obtenidos tras la aplicación de la metodología planteada.

\section{Análisis topográfico}

Se observó en la plancha 1:25000 el punto donde descarga sus aguas el Embalse del Sisga. A partir de este hallazgo se determinó por curvas de nivel la mayor caída de agua con el fin de identificar el aprovechamiento óptimo de energía potencial y establecer el recorrido de la tubería que transportará el agua a la casa de máquinas. La cota de salida del agua del embalse está a una altura de $2.944,29 \mathrm{~m} \mathrm{~s}$. n. m., mientras que la cota donde terminaría la tubería de transporte y la descarga de aguas a nivel del río Bogotá se encuentra a una altura de $2.600 \mathrm{~m} \mathrm{~s}$. n. m., lo que por diferencia representa una caída bruta de agua de $344,29 \mathrm{~m}$. Por otra parte, la longitud del recorrido de la tubería (figura 3) se calculó por medio de la escala del mapa, dando como resultado una longitud de $3.125 \mathrm{~m}$. Esta información resulta importante para determinar las pérdidas por fricción en el sistema.

Figura 2. Recorrido de la tubería en el sistema

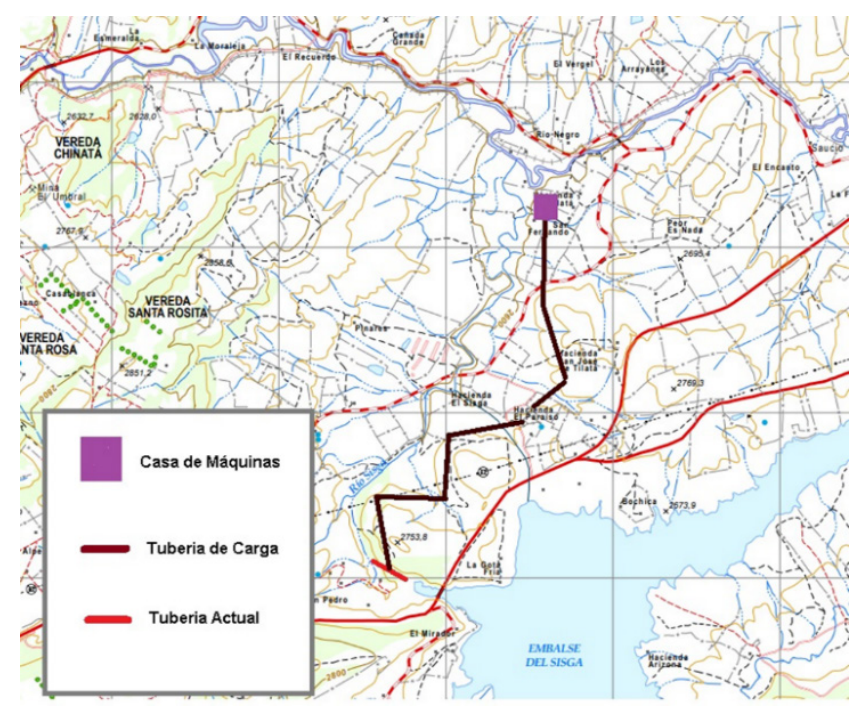

Fuente: elaboración propia.

\section{Evaluación del recurso hidroenergético [T2]}

Tras realizar la curva de duración de caudales se logró determinar el caudal medio anual que descarga el embalse, el cual equivale a $2,77 \mathrm{~m}^{3} / \mathrm{s}$, valor seleccionado 
como caudal de diseño. La figura 3 representa la CDC para la descarga del embalse, donde se puede apreciar la frecuencia acumulada de ocurrencia del caudal determinado. La tabla 2 muestra los caudales correspondientes a diferentes frecuencias, datos obtenidos de la CDC.

Figura 3. Curva de duración del caudal del Embalse del Sisga

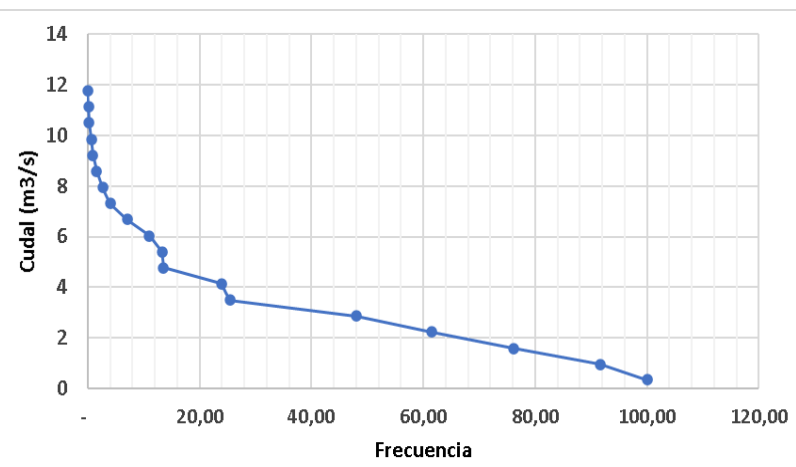

Fuente: elaboración propia.

Tabla 2. Frecuencias de diferentes caudales del Embalse del Sisga

\begin{tabular}{|c|c|}
\hline Frecuencia & Caudal $(\mathrm{m} 3 / \mathbf{s})$ \\
\hline $100 \%$ & 0,35 \\
\hline $95 \%$ & 0,7 \\
\hline $50 \%$ & 2,77 \\
\hline $5 \%$ & 7,1163 \\
\hline
\end{tabular}

Fuente: elaboración propia.

A partir del desarrollo de la CDC fue posible realizar una curva de duración de potencias (CDP). Este análisis permite determinar el caudal que aporta mayor energía al sistema (figura 4).

Figura 4. Curva de duración de potencia

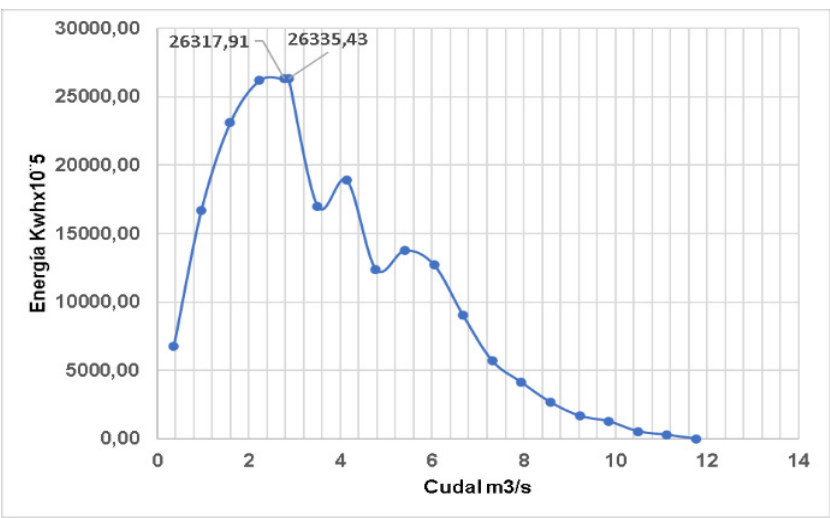

Fuente: elaboración propia.
En la figura 4 se aprecia que la máxima energía que podría producirse es de $28.115,75 \mathrm{Kwh} \times 10^{-5}$, resultado de un caudal de $2,86 \mathrm{~m}^{3} / \mathrm{s}$. El valor de $28.096,95 \mathrm{kWh} \times 10$ corresponde a la energía que puede producir el caudal medio calculado en la CDC, valor tomado como caudal de diseño $2,77 \mathrm{~m}^{3} / \mathrm{s}$ (Florez, 2011). De otro lado, es posible analizar la CDP relacionando la frecuencia del caudal con la potencia en kW, como se aprecia en la figura 5.

Figura 5. CDP potencia vs. frecuencia

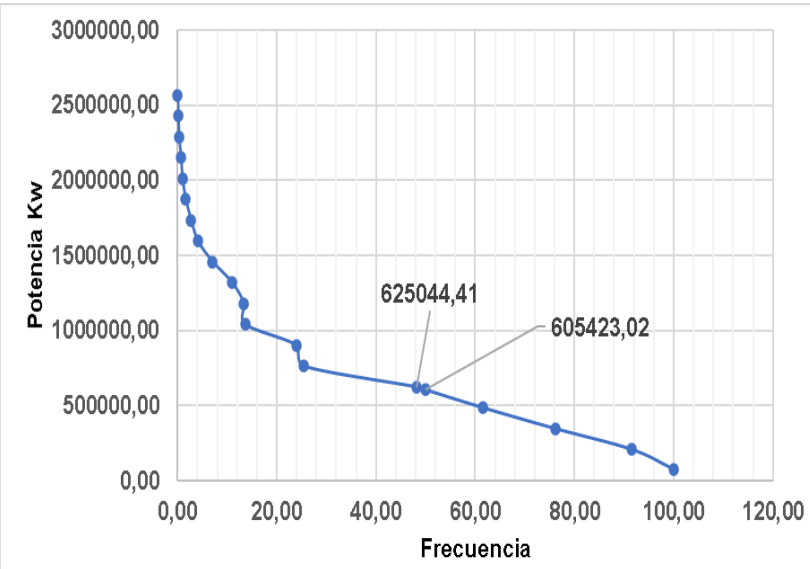

Fuente: elaboración propia.

Al relacionar la potencia y la frecuencia de ocurrencia del caudal se puede apreciar que con un rendimiento de $48 \%$ $\left(2,86 \mathrm{~m}^{3} / \mathrm{s}\right)$ la potencia equivale a $670.707,62 \mathrm{~kW}$, mientras que para $50 \%\left(2,77 \mathrm{~m}^{3} / \mathrm{s}\right)$ corresponde a $649.541,91$ $\mathrm{kW}$, equivalente al caudal de diseño. Vale la pena resaltar que los datos de las curvas se calculan a partir de un rendimiento de $92 \%$ y una altura neta 342,52 m. Estos porcentajes de potencia son producidos en un año.

\section{Selección de componentes hidráulicos y electromecánicos}

La tabla 3 presenta los principales parámetros de diseño. Estos se calcularon durante el desarrollo de la investigación y son de gran importancia para establecer las características de los componentes del sistema.

Tabla 3. Parámetros de dimensionamiento

\begin{tabular}{|l|l|}
\hline Caudal de diseño & $2,77 \mathrm{~m}^{3} / \mathrm{s}$ \\
\hline Longitud de la tubería & $3.125 \mathrm{~m}$ \\
\hline Perdidas por fricción & $23,44 \mathrm{~m}$ \\
\hline Caída bruta & $344,29 \mathrm{~m}$ \\
\hline Caída neta & $320,85 \mathrm{~m}$ \\
\hline
\end{tabular}

Fuente: elaboración propia. 


\section{Tubería de carga}

Con los principales parámetros establecidos en los anteriores estudios fue posible determinar el diámetro mínimo que debería tener la tubería, dando como resultado un valor de $0,70 \mathrm{~m}$, teniendo en cuenta que el material seleccionado fue el acero. Este diámetro permite que el sistema funcione en condiciones estables. En la figura 6 se puede apreciar la evaluación del diámetro económico para las condiciones del proyecto.

Figura 6. Evaluación del diámetro económico

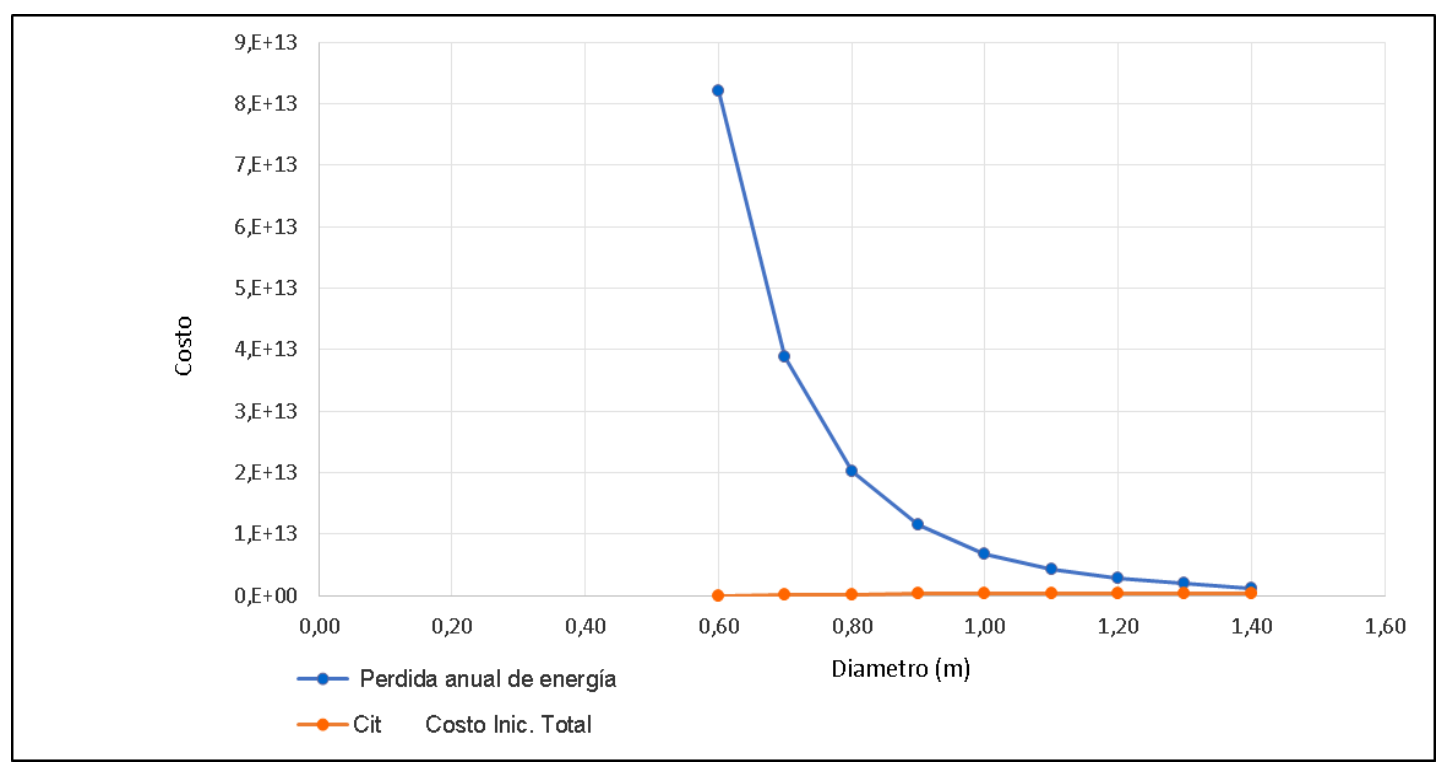

Fuente: elaboración propia.

En la figura 6 se observa la relación entre las pérdidas de energía y los costos para una tubería en acero de diferentes diámetros. La relación inversa entre costo de tubería y pérdidas de energía es notable, por lo que el dato que mejor se ajusta a las necesidades del proyecto es el de 1,40 m. Este valor corresponde al diámetro que genera las menores pérdidas de energía y el menor costo por valor de materiales. Vale la pena resaltar que para un diámetro de 1,40 $\mathrm{m}$ y las condiciones del fluido la velocidad es de 1,8 $\mathrm{m} / \mathrm{s}$, la cual no representa un valor que pueda llegar a generar complicaciones en la operatividad de la PCH.

Finalmente, se opta por promediar el diámetro obtenido en este análisis $(1,40 \mathrm{~m})$ y el diámetro mínimo calculado anteriormente en los parámetros para una tubería de acero $(0,70 \mathrm{~m})$. De esta forma, el diámetro que mejor se ajusta es de $1,05 \mathrm{~m}$.

\section{Casa de máquinas}

Para el generador se seleccionaron variables que determinaran el mejor rendimiento del sistema. En la tabla 4 se muestran los valores que se deben tener en cuenta para la selección del generador.

Tabla 4. Datos del generador

\begin{tabular}{|l|l|}
\hline Eficiencia & 0,95 \\
\hline Coseno Fi (Grados) & 0,8 \\
\hline Frecuencia (Ciclos) & 60 \\
\hline Pares de polos & 4 \\
\hline Velocidad real de rotación (RPM) & 514 \\
\hline Diámetro interior Dint (cm) & 456,98 \\
\hline Diámetro exterior Dext $(\mathrm{cm})$ & 584,89 \\
\hline Altura del generador $\mathrm{Hg}(\mathrm{cm})$ & 305 \\
\hline
\end{tabular}

Fuente: elaboración propia. 
La turbina seleccionada para este proyecto fue tipo Francis, equipo que sirve para trabajar con alturas medias de más de 100 metros y caudales de hasta 30 $\mathrm{m}^{3} / \mathrm{s}$. La turbina tipo Francis es catalogada como de reacción, flujo mixto, centrípeto y de admisión total. Sus principales ventajas son las bajas pérdidas hidráulicas, alta eficiencia, alta velocidad específica y bajos costos en acoplamiento con el generador. Este equipo opera a grandes rangos de alturas y caudales. En la figura 7 se puede apreciar el esquema general de instalación de una turbina Francis (Morales et al., 2014). La tabla 5 describe los principales datos de la turbina.

Figura 7. Esquema general de instalación de una turbina Francis

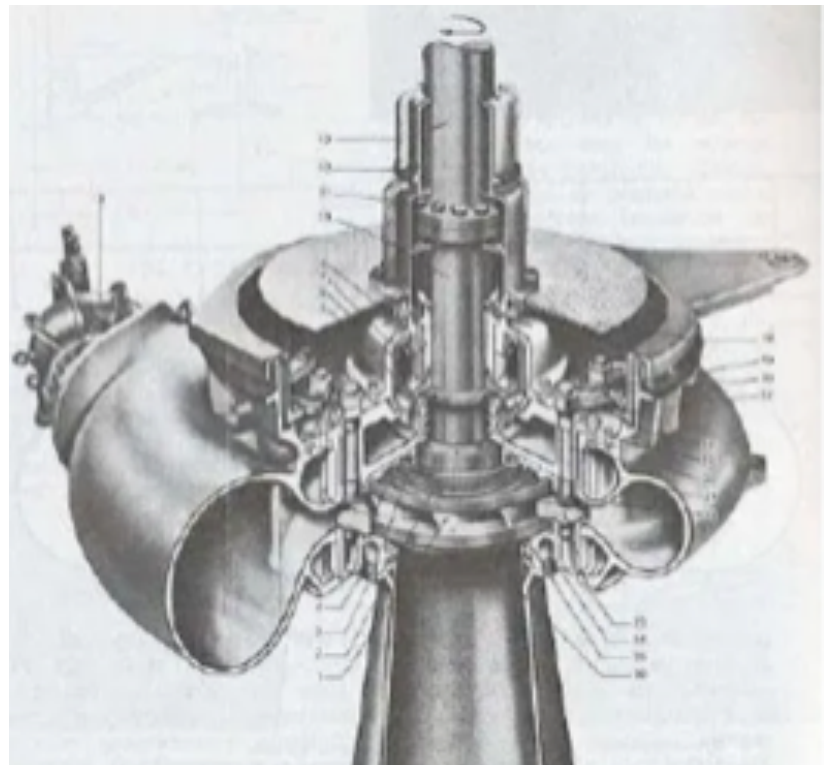

Fuente: Ayala et al. (2015).

Tabla 5. Datos de la turbina tipo Francis para el sistema

\begin{tabular}{|l|l|}
\hline Tipo & Francis \\
\hline Eficiencia nt & 0,92 \\
\hline Diámetro del Rotor $(\mathrm{m})$ & 1,02 \\
\hline Velocidad del Rotor Vt (rpm) & 1130 \\
\hline Rapidez de la Turbina ns (rpm) & 87 \\
\hline Rotación Especifica nq & 26 \\
\hline
\end{tabular}

Fuente: elaboración propia.

La turbina cuenta con un tubo de descara cónico vertical y un tubo de descarga arrodillado. Las dimensiones de estas estructuras se muestran en las tablas 6 y 7. Los datos calculados para el generador y la turbina, junto con sus partes, son de gran importancia para entender el dimensionamiento de los equipos necesarios en el sistema.

Tabla 6. Datos del tubo de descarga cónico vertical

\begin{tabular}{|l|l|}
\hline Ángulo de ampliación B & $6^{\circ}$ \\
\hline Diámetro de entrada De $(\mathrm{m})$ & 1,02 \\
\hline Diámetro de salida DS $(\mathrm{m})$ & 1,89 \\
\hline Longitud calculada $(\mathrm{m})$ & 4,09 \\
\hline Velocidad a la salida del rotor $(\mathrm{m} / \mathrm{s})$ & 3,36 \\
\hline Velocidad a la salida del tubo $(\mathrm{m} / \mathrm{s})$ & 0,99 \\
\hline
\end{tabular}

Fuente: elaboración propia.

Tabla 7. Datos del tubo de descarga arrodillado

\begin{tabular}{|l|l|}
\hline Sección del cuello en la altura $(\mathrm{m})$ & 1,02 \\
\hline Sección de salida en la altura $(\mathrm{m})$ & 1,28 \\
\hline Tubo de descarga en la altura $\mathrm{Ht}(\mathrm{m})$ & 2,66 \\
\hline Longitud de la parte horizontal $\mathrm{Lh}(\mathrm{m})$ & 5,12 \\
\hline Ancho del tubo arrodillado At $(\mathrm{m})$ & 3,74 \\
\hline Profundidad de sumergencia $\mathrm{Hs}(\mathrm{m})$ & $-102,94$ \\
\hline Velocidad en la cámara Vc (m/s) & 10,2 \\
\hline
\end{tabular}

Fuente: elaboración propia.

\section{Análisis energético del sistema}

El desarrollo del proyecto estimó la generación de energía por medio de diferentes cálculos, teniendo en cuenta la utilización de dos turbinas. En la tabla 8 se pueden apreciar las características de la generación.

Tabla 8. Generación de energía y potencia del sistema

\begin{tabular}{|c|c|}
\hline & Dos turbinas \\
\hline Caudal neto & $2,77 \mathrm{~m} 3 / \mathrm{s}$ \\
\hline Cabeza estática & $344,29 \mathrm{~m}$ \\
\hline Pérdidas en la tubería & $23,44 \mathrm{~m}$ \\
\hline Cabeza útil & $320,85 \mathrm{~m}$ \\
\hline Potencia máxima & $7.628,85 \mathrm{~kW}$ \\
\hline Potencia media & $7.438,11 \mathrm{~kW}$ \\
\hline Potencia firme & $3.984,23 \mathrm{~kW}$ \\
\hline Factor de utilización & $97 \%$ \\
\hline Generación media anual & $65.157,85$ miles $\mathrm{kWh}$ \\
\hline Generación firme anual & $34.901,89$ miles $\mathrm{kWh}$ \\
\hline
\end{tabular}

Fuente: elaboración propia. 
Teniendo en cuenta que se seleccionan dos turbinas tipo Francis, se obtienen los datos presentados en la tabla 8. Cada turbina trabajará con un caudal de 1,38 $\mathrm{m}^{3} / \mathrm{s}$ y a su vez puede generar una potencia máxima de 3.814,42 kW, alcanzando hasta $7.628,85 \mathrm{~kW}$. La potencia media con la que trabajará normalmente la casa de máquinas es de 7.438,11 kW. Por último, la potencia firme, que hace referencia a la operación con un caudal bajo, es de $3.984,23 \mathrm{~kW}$.
La tabla 9 describe el comportamiento de generación de cada turbina. En esta tabla se puede observar que la turbina 1 tiene mayor rendimiento de generación, dado que a la turbina 2 ingresa un menor caudal. No obstante, el rendimiento de ambos equipos es óptimo. La generación media anual de potencia, que dio como resultado $65.157,85 \mathrm{kWh}$, es un dato importante dado que con este valor será posible evaluar el impacto ambiental positivo de este proyecto.

Tabla 9. Cálculo de la generación de energía

\begin{tabular}{|c|c|c|c|c|c|c|c|c|c|}
\hline \multirow[b]{2}{*}{$\begin{array}{c}\text { Duración } \\
\text { (1) } \\
\%\end{array}$} & \multirow[b]{2}{*}{$\begin{array}{l}\text { Caudal } \\
\text { río } \\
\left(\mathrm{m}^{3} / \mathrm{s}\right)\end{array}$} & \multicolumn{4}{|c|}{ 1a turbina } & \multicolumn{3}{|c|}{ 2a turbina } & \multirow[b]{2}{*}{$\begin{array}{c}\text { Energía } \\
\text { generada } \\
\text { (miles } \\
\text { kWh) }\end{array}$} \\
\hline & & $\begin{array}{l}\text { Caudal } \\
\text { turbina } \\
\left(\mathrm{m}^{3} / \mathrm{s}\right)\end{array}$ & $\begin{array}{l}\text { Admi- } \\
\text { sión } \\
(\%)\end{array}$ & $\begin{array}{c}\text { Rendimien- } \\
\text { to } \\
(2) \\
\%\end{array}$ & $\begin{array}{c}\text { Energía } \\
\text { generada } \\
\text { (miles kWh) }\end{array}$ & $\begin{array}{c}\text { Caudal } \\
\text { turbina } \\
\left(\mathrm{m}^{3} / \mathrm{s}\right)\end{array}$ & $\begin{array}{c}\text { Admisión } \\
(\%)\end{array}$ & $\begin{array}{c}\text { Rendimiento } \\
\text { (\%) }\end{array}$ & \\
\hline 100 & 0,250 & 0,25 & 18 & 72 & 50 & 0,00 & 0 & 65 & 0 \\
\hline 99 & 0,950 & 0,95 & 69 & 72 & 189 & 0,00 & 0 & 68 & 0 \\
\hline 98 & 1,580 & 1,39 & 100 & 72 & 276 & 0,19 & 14 & 70 & 37 \\
\hline 97 & 2,860 & 1,39 & 100 & 92,5 & 355 & 1,39 & 100 & 70 & 267 \\
\hline 96 & 3,490 & 1,39 & 100 & 92,5 & 355 & 1,39 & 100 & 70 & 267 \\
\hline 95 & 4,130 & 1,39 & 100 & 92,5 & 1773 & 1,39 & 100 & 70 & 1337 \\
\hline 90 & 4,780 & 1,39 & 100 & 92,5 & 1773 & 1,39 & 100 & 70 & 1337 \\
\hline 85 & 4,130 & 1,39 & 100 & 92,5 & 1773 & 1,39 & 100 & 70 & 1337 \\
\hline 80 & 4,780 & 1,39 & 100 & 92,5 & 1773 & 1,39 & 100 & 70 & 1337 \\
\hline 75 & 5,400 & 1,39 & 100 & 92,5 & 1773 & 1,39 & 100 & 90 & 1718 \\
\hline 70 & 6,040 & 1,39 & 100 & 92,5 & 1773 & 1,39 & 100 & 90 & 1718 \\
\hline 65 & 6,670 & 1,39 & 100 & 92,5 & 1773 & 1,39 & 100 & 90 & 1718 \\
\hline 60 & 7,310 & 1,39 & 100 & 92,5 & 1773 & 1,39 & 100 & 90 & 1718 \\
\hline 55 & 7,940 & 1,39 & 100 & 92,5 & 1773 & 1,39 & 100 & 90 & 1718 \\
\hline 50 & 7,940 & 1,39 & 100 & 92,5 & 1773 & 1,39 & 100 & 90 & 1718 \\
\hline 45 & 8,580 & 1,39 & 100 & 92,5 & 1773 & 1,39 & 100 & 90 & 1718 \\
\hline 40 & 8,580 & 1,39 & 100 & 92,5 & 1773 & 1,39 & 100 & 90 & 1718 \\
\hline 35 & 9,220 & 1,39 & 100 & 92,5 & 1773 & 1,39 & 100 & 92,5 & 1766 \\
\hline 30 & 9,220 & 1,39 & 100 & 92,5 & 1773 & 1,39 & 100 & 92,5 & 1766 \\
\hline 25 & 9,800 & 1,39 & 100 & 92,5 & 1773 & 1,39 & 100 & 92,5 & 1766 \\
\hline 20 & 10,490 & 1,39 & 100 & 92,5 & 1773 & 1,39 & 100 & 92,5 & 1766 \\
\hline 15 & 11,760 & 1,39 & 100 & 92,5 & 1773 & 1,39 & 100 & 92,5 & 1766 \\
\hline 10 & 11,760 & 1,39 & 100 & 92,5 & 3545 & 1,39 & 100 & 92,5 & 3532 \\
\hline Energía anual & \multicolumn{5}{|c|}{34.902 kWh } & \multicolumn{4}{|c|}{$32.029 \mathrm{kWh}$} \\
\hline \multicolumn{2}{|l|}{$\begin{array}{c}\text { Potencia } \\
\text { media }\end{array}$} & \multicolumn{4}{|c|}{$3.984 \mathrm{~kW}$} & \multicolumn{4}{|c|}{3.656 kW } \\
\hline $\begin{array}{l}\text { Factor } \\
\text { utilización }\end{array}$ & & \multicolumn{4}{|c|}{$104 \%$} & \multicolumn{4}{|c|}{$96 \%$} \\
\hline
\end{tabular}




\section{Análisis ambiental}

Se calcularon las toneladas de $\mathrm{CO}_{2}$ anuales para la generación de electricidad de este proyecto, obteniendo que al producir $6.515 \mathrm{MW}$ por medio de una $\mathrm{PCH}$ se dejarían de producir 2.391 t $\mathrm{CO}_{2}$ generadas por la quema de combustibles fósiles en una central termoeléctrica. En la tabla 10 se pueden observar los valores correspondientes a la generación de energía anual y su relación con la producción de t de $\mathrm{CO}_{2}$.

Tabla 10. Producción de energía y ahorro de $\mathrm{CO}_{2}$

\begin{tabular}{|c|c|c|c|}
\hline Años & kWh & GWh & MWh \\
\hline $3-5$ & 65.158 .000 & 65.158 & $6.515,8$ \\
\hline $6-10$ & 65.158 .000 & 65.158 & $6.515,8$ \\
\hline $11-15$ & 65.158 .000 & 65.158 & $6.515,8$ \\
\hline $16-20$ & 65.158 .000 & 65.158 & $6.515,8$ \\
\hline $21-25$ & 65.158 .000 & 65.158 & $6.515,8$ \\
\hline $25-30$ & 65.158 .000 & 65.158 & $6.515,8$ \\
\cline { 1 - 2 } 0.367 & Total & 1 & MWh \\
\hline 2391.005 & $\mathbf{t ~ C O 2}$ & 6515 & $\mathbf{M W}$ \\
\hline
\end{tabular}

Fuente: elaboración propia.

\section{CONCLUSIONES}

En el presente estudio se determinaron diferentes parámetros para evaluar la viabilidad de instalar una pequeña central hidroeléctrica en el Embalse del Sisga, aprovechando el caudal de descarga de este cuerpo de agua.

El análisis topográfico determinó el recorrido más conveniente de la tubería, evidenciando pérdidas por fricción considerables. Sin embargo, gracias a la alta caída del agua (344,29 m), el potencial energético se vio beneficiado por esta diferencia entre cotas.

El análisis hidrológico determinó el caudal de diseño y la capacidad energética del mismo. Esta investigación reveló el buen rendimiento del caudal, arrojando una capacidad de producir 649.541,91 kW anuales, con un rendimiento aproximado de 92 \%. Estos resultados hacen que la viabilidad del proyecto sea positiva, dado que para una $\mathrm{PCH}$ son valores altos de producción. Además, se evidencia que es posible aprovechar el recurso hídrico del embalse sin afectar los otros usos que la población hace de este.

Al seleccionar la tubería se procuró mantener los menores costos posibles. Por ello, se seleccionó el acero y se determinó el diámetro económico, disminuyendo las pérdidas energéticas y aumentando el ahorro monetario en la instalación de este tipo de proyectos. El estudio muestra que es factible realizar el proyecto sin generar grandes costos por cuenta de la instalación de la tubería.

Se determinó que la producción energética tiende a ser óptima durante el funcionamiento del sistema a lo largo del tiempo. Esto se debe a que incluso en época de sequía la PCH produciría hasta $34.901,89$ kWh como generación firme anual, cifra óptima para una pequeña central con estas características. Esto ayudaría a suplir la demanda energética de otras centrales con problemas de abastecimiento.

Dado que la $\mathrm{PCH}$ en el embalse no requiere de muchas estructuras y además aprovecha el recurso hídrico de un cuerpo de agua ya represado, esta no representa cambios significativos para la fauna y flora del área de estudio ni genera impactos negativos en el componente sociocultural de la zona. Por el contrario, el proyecto traería impactos positivos, como una mayor cobertura en el servicio de energía eléctrica y una disminución en la generación de GEl, como se mostró en los resultados del estudio ambiental, generando un ahorro de un poco más de $2.390 \mathrm{t}$ de $\mathrm{CO}_{2}$

\section{AGRADECIMIENTOS}

Los autores agradecen al Grupo de Investigación TECNOAMBIENTAL y a la Universidad Libre por su colaboración en el desarrollo de la investigación. También agradecen a la Corporación Autónoma Regional de Cundinamarca (CAR) por suministrar información relevante para alcanzar los objetivos de la investigación. Este trabajo es dedicado a la memoria del ingeniero Jesús Ernesto Torres Quintero.

\section{REFERENCIAS}

Asociación Colombiana de Generadores de Energía Eléctrica [Acolgen]. (2019). Acolgen. https://www. acolgen.org.co/ 
Ayala, D., Chaverrea, M., Constante, D., Mosquera, D., Navarrete, K., Ramírez, M., Velastegui, S., \& Zagal, J. (2015). Turbina Francis. Escuela Politécnica Nacional.

Comisión Nacional de Riego. (2020). Manual de diseño de proyectos microhidroeléctricos en obras de riego. Impresores S. A.

Consorcio Ecoforest Ltda. (2018). Elaboración del diagnóstico, prospectiva y formulación de la cuenca hidrográfica del río Bogotá subcuenca Embalse Sisga. Consorcio Ecoforest Ltda.

Corporación Autónoma Regional de Cundinamarca [CAR]. (2020). Manual de operaciones del Embalse del Sisga. CAR.

Fundación Solar. (2018). United Nations Development Programme. Centrales hidroeléctricas de pequeña escala, la experiencia de la Fundación Solar en Guatemala. Fundación Solar. https:// www.undp.org/content/dam/guatemala/06\%20 Centrales\%20Hidroel\%c3\%a9ctricas\%20 peque\%c3\%b1a\%20escala.pdf

Grupo Intergubernamental de Expertos sobre el Cambio Climático [IPCC]. (2011). Fuentes de energía renovables y mitigación del cambio climático: Unidad de apoyo técnico del Grupo de trabajo III, Instituto de investigación sobre el impacto del clima Potsdam, 2011. IPCC.

Gugler Technology for Hydropower Plants (2020, diciembre 14). Gugler Water Turbines $\mathrm{GmbH}$. https://www.gugler.com/es/

Instituto de Ciencias Nucleares y Energías Alternativas. (1997). Guía para diseño de pequeñas centrales hidroeléctricas. Ministerio de Minas y Energía.
Morales, S., Corredor, L., Paba, J., \& Pacheco, L. (2014). Etapas de desarrollo de un proyecto de pequeñas centrales hidroeléctricas: contexto y criterios. Dyna, 81(184), 178-185. https://doi. org/10.15446/dyna.v81n184.39757

Organización Colparques. (2020, agosto 5). Embalse del Sisga. http://www.colparques.net/SISGA

Organización de las Naciones Unidas Para el Desarrollo Industrial [ONUDI]. (2019). Informe mundial sobre el desarrollo de la pequeña central hidroeléctrica. ONUDI.

Ortiz-Flórez, R. (2011). Pequeñas centrales hidroeléctricas. Ediciones de la U.

Torres, E. (2010). Investigación en pequeñas centrales en Colombia. Ingenio Libre, 1(2), 1-15.

Unidad de Planeación Minero-Energética (UPME). (2020). Estudio de generación eléctrica bajo escenario de cambio climático. UPME.

United Nations Industrial Development Organization [UNIDO]. (2016). World small hydropower development report. UNIDO.

Vélez-Álvarez, L. G. (2020). Colombia: el futuro de las pequeñas centrales hidroeléctricas. https://panampost. com/luis-guillermo-velez/2020/02/17/ colombia-centrales-hidroelectricas/

Yunus, C., \& Cimbala, J. (2006). Mecánica de fluidos fundamentos y aplicaciones. McGraw-Hill Interamericana. 